\title{
A Generic Approach for Weight Assignment to the Decision Making Parameters
}

\author{
Md. Zahid Hasan ${ }^{1}$, Shakhawat Hossain ${ }^{2}$, Mohammad Shorif Uddin ${ }^{3}$, Mohammad Shahidul Islam ${ }^{4}$ \\ Department of Computer Science and Engineering Jahangirnagar University, Dhaka, Bangladesh ${ }^{1,3}$ \\ Department of CSE, International Islamic University Chittagong, Chattogram, Bangladesh ${ }^{2}$ \\ Institute of Information Technology, Jahangirnagar University, Dhaka, Bangladesh ${ }^{4}$
}

\begin{abstract}
Weight assignment to the decision parameters is a crucial factor in the decision-making process. Any imprecision in weight assignment to the decision attributes may lead the whole decision-making process useless which ultimately mislead the decision-makers to find an optimal solution. Therefore, attributes' weight allocation process should be flawless and rational, and should not be just assigning some random values to the attributes without a proper analysis of the attributes' impact on the decision-making process. Unfortunately, there is no sophisticated mathematical framework for analyzing the attribute's impact on the decision-making process and thus the weight allocation task is accomplished based on some human sensing factors. To fill this gap, present paper proposes a weight assignment framework that analyzes the impact of an attribute on the decision-making process and based on that, each attribute is evaluated with a justified numerical value. The proposed framework analyzes historical data to assess the importance of an attribute and organizes the decision problems in a hierarchical structure and uses different mathematical formulas to explicit weights at different levels. Weights of mid and higherlevel attributes are calculated based on the weights of root-level attributes. The proposed methodology has been validated with diverse data. In addition, the paper presents some potential applications of the proposed weight allocation scheme.
\end{abstract}

Keywords-Multiple attribute decision problem; average term frequency; cosine similarity; weight setup for multiple attributes; decision making

\section{INTRODUCTION}

In decision-making approaches, the decision-makers need to obtain the optimal alternative from a set of predefined alternatives based on some decision parameters say attributes. Attribute's weight states the relative importance of an attribute and is numerically described to address the impact of an attribute on the decision-making process. A precise decisionmaking process mostly depends upon its attributes' weights. Decision attributes in decision problems can be organized in a hierarchical layer. The root-layer attributes of a decision problem expose the basic decision parameters whereas intermediate-layer attributes have a significant dependency on the root-layer attributes.

However, all the attributes in a decision solution are not equally important. So, to identify the importance of an attribute relative to other attributes in decision problem solution, a weight is assigned to each attribute. For example, suppose, a music school determines to appoint a music teacher and for that, the school committee sets two basic quality measurement attributes- knowledge on a musical instrument (KMI) and knowledge on geography (KG). However, the case may happen that, a candidate scores 120 marks (40 marks in $\mathrm{KMI}$ and 80 marks in KG) out of 200 where another candidate obtains 100 marks (70 in KMI and 30 marks in KG). If each of these two attributes is given similar importance then the first candidate will be selected which is by no means, an optimal decision. So, it is important to set a specific weight to each attribute in a decision-making process. But there is no mathematical approach that helps the decision-makers to allocate weights to the decision parameters. Decision-makers very often depend on domain experts to determine attributes' weights manually which produces some uncertainties in the decision-making process and consequently, leads to a nonoptional decision. In real-life scenarios, a decision-maker himself sets an identical weight to each decision-making attribute rather than depending on domain experts which in consequence makes the decision-making framework find a troublesome decision solution.

However, this paper proposes a very straightforward formula to allocate weights to the decision attributes. The paper proposes the Term Frequency to allocate weights to the root level attributes and the Cosine Similarity to generate weights for the intermediate level attributes. Both approaches analyze some historical data to emerge the weights of the decision attributes.

The paper is organized as follows: Section II provides a detailed description of the existing weight allocation methodologies. Based on the study of related works, a new generic weight assignment methodology is proposed in Section III. In Section IV, a numerical experiment on the proposed methodology is provided. The system is validated in Section V through the representation of its results at different critical situations. The paper is concluded in Section VI.

\section{RELATED WORKS}

In multiple attribute decision-making problems, the relative importance of each attribute allocated by an expert has a great impact to evaluate every alternative [1]. The multiple attribute decision-making (MADM) method offers a practical and efficient way to obtain a ranking of all the alternatives based on non-relative and inconsistent attributes [2]. For that reason, it is especially imperative to find a logical and sensible weight allocation scheme. In real life, the imperfect, inexact information and the impact of particular and individual preference lead to expanding the indecision and trouble in 
weight calculation and distribution in the decision-making problem [3].

The decision scientists have proposed various methodologies for obtaining attributes' weights of the decision-making problems. The present methods can be generally divided into three groups: subjective, objective and integrated approach [4]. In the subjective approach, the decision-makers set the attributes' weights by using their preference knowledge [5]. AHP [6] and Delphi method [7] are the standard methods for determining subjective weights based on the preference of decision-makers. Ranked and point allocation methods proposed by Doyle et al [8] and rank order distribution method provided by Roberts and Goodwin [9] are also some subjective weighting approaches.

In the objective approach, the decision-makers set the attribute-weights by using applicable facts, rational implications, and viewpoints. Entropy-based method [10], TOPSIS method [11] and mathematical programming based method [12] follow the objective approach to allocate weights to the decision parameters. Decision-makers' preferences and evidence-facts of the specific problem are considered simultaneously in the integrated approach for obtaining the attributes-weights. Cook and Kress proposed a preferenceaggregation model [13] which is actually an integrated approach for allocating weights to the decision attributes. Fan et al. [14], Horsky and Rao [15], and Pekelman and Sen [16] also constructed some optimization-based models.

However, it is crucial to select suitable attributes' weights in decision-making conditions since the diverse values of attributes' weights may effect in unlike ranking order of alternatives. Nevertheless, in most MADM scenarios, the preference of the attributes over the alternatives distributed by decision-makers is typically not adequate for the crisp numerical data, because things are uncertain, fuzzy and possibly inclined by the subjectivity of the decision-makers, or the knowledge and data about the problem domain are insufficient during the decision-making the process.

Based on the above analysis, many factors affect the weight allocation, there should be considered that the importance of attribute is reflected by the objective data and the subjective preference of the decision-makers. But there is no better method to fuse the subjective and objective weights in existing MADM literature. With the motivation of establishing a weight allocation approach in a uniform as well as effective way, this paper proposes the current generic scheme.

\section{METHODOLOGY}

\section{A. Problem Statement}

Decision-makers use different methodologies to capture different types of decision problems. Different methodologies calculate alternatives' scores in different ways. However, almost all the available advanced decision-making approaches set some specific weights to distinguish the decision-making parameters based on their relative importance on the decisionmaking process. Based on the decision-making process and decision approach, the attributes' weights setup process can be categories into the following classes.

1) The rule-based decision-making processes set a random weight for each of its attributes. Some rule-based approaches like the Evidential Reasoning (ER) approach use those random values to calculate the final result. On the other hand, some advance decision-making approaches update the primarily set up random weights to enhance the decision-making accuracy. For example, the RIMER approach updates the activation weight to emerge an accurate decision result.

2) Decision-making approaches like deep learning methodologies use some random values as its primary activation weight. For example, ANN, RNN, CNN or GAN use some random values to activate their attributes' weights. Later, these approaches update the weights to reach the maximum accuracy in any type of decision-making process. So, in terms of decision attributes' weight generation, decision problems of the existing decision-making approaches can be listed as:

a) Rule-based decision-making approaches use some random values to update its decision attributes' weights which lead the decision-making approaches to some troublesome results.

b) Weight updating processes used in decision-making approaches are not enough matured and as a consequence decision-making approaches very often fail to reveal the decision results with enough confidence.

c) Deep learning approaches take huge time to update its activation weights as it requires multiple iterations to update attributes weights.

The above discussion makes it clear that decision approaches need to follow an appropriate weight setup process in order to generate an accurate decision result under any unfair circumstance like risk or uncertainties.

\section{B. Problem Structuring}

Based on types and nature, decision problems can be classified into two basic classes:

- Single-layer decision problems; and

- Multilayer decision problems.

In single-layer decision problems, decision solutions are made with the direct involvement of the decision attributes. Only the basic decision parameters that have a direct impact on decision problem solutions construct the single -layer decision architecture. This type of attribute can be represented using the architecture (see Fig. 1).

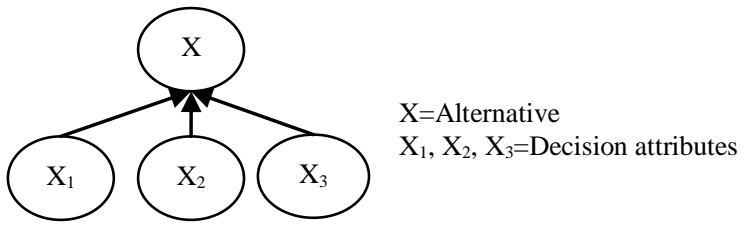

Fig. 1. Single Layer Decision Problem. 


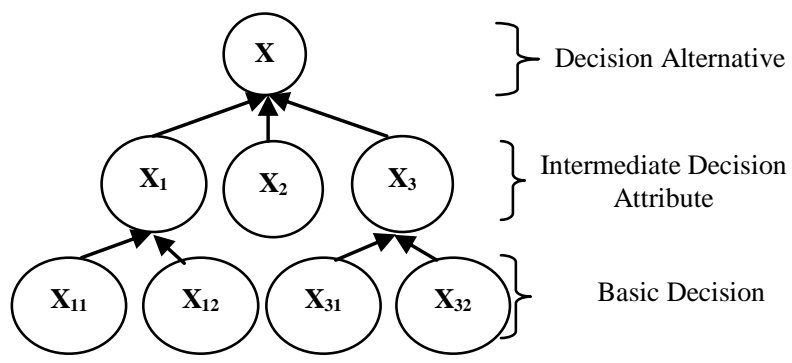

Fig. 2. Multi Layer Decision Problem.

On the other hand, multi-level decision problems depend on one or more intermediate levels to make the final decision rather than just depending on the basic attributes. These types of decision problems can be expressed by an Architectural Theory Diagram (ATD) or a hierarchical structure (see Fig. 2).

So, weight generation for decision attributes can necessarily be associated at root level or at both root and intermediate levels of the decision problem architecture.

\section{Weight Generation for Root-layer Attributes}

Weights for the basic attributes of a decision problem can be generated by analyzing the historical data. This paper proposes the Term Frequency (TF) as the data analyzing tool for weight assignment to the decision attributes. Term frequency is a very sophisticated technique for revealing the importance of a term for a specific purpose. Term frequency assigns weight to an attribute based on the number of times an attribute appears in a dataset. Term frequency of a term ' $t$ ' can mathematically represented as

Term Frequency, $T F=\frac{f_{t, d}}{\sum_{t \prime \in d} f_{t \prime, d}}$

However, very often weight estimation of an attribute becomes troublesome because of different TF from different datasets. In that case, this paper proposes a normalized term frequency to expose the importance of an attribute. The normalization task is accomplished by calculating the average of the Term frequencies measured from different datasets. The average term frequency of an attribute can be represented as:

$\operatorname{AvgTF_{X}}=\frac{\sum_{i=1}^{N} T F_{x}}{L}$

Here, $A v g T F_{X}$ refers to an average term frequency. The average TF of an attribute denotes the actual weight of an attribute.
WeightofattributeX $=\frac{\left(T F_{x 1}+T F_{x 2}+T F_{x 3}+\ldots+T F_{x n}\right)}{n}$

Here, $X=\left\{X_{1}, X_{2}, X_{3}, \ldots, X_{n}\right\}$ denotes the value of attributes and $\mathrm{n}$ is the total number of attributes. So, the average TF will be considered as the final weights of the rootlevel attributes.

\section{Different Cases of Weight Generation using Term Frequency}

Weight generation by analyzing the historical data can be categorized into two basic classes based on the data types.

1) Weight calculation from textual data: In case of textual datasets, the weight of an attribute can be assigned based on the times the attributes appears in each document. Thus, the weights of an attribute in different datasets are calculated. Finally, the average weight can be measured. For example, Table I shows the weight calculation process from two different documents.

The term frequency of "Dhaka" for each of these documents can be calculated as:

$T F($ "Dhaka", $d 1)=\frac{3}{22}=0.136$ and

$T F($ "Dhaka", $d 2)=\frac{5}{20}=0.25$

Where, $d l$ and $d 2$ both are the two separate documents. So, the average $T F$ or the actual weight of "Dhaka" is calculated by using equation 3 .

AvgTF $=0.386$

TABLE. I. CALCULATION OF TERM FREQUENCY FROM TEXT

\begin{tabular}{|l|l|l|l|}
\hline \multicolumn{2}{|l|}{ Document 1 $(\boldsymbol{d} 1)$} & \multicolumn{2}{l|}{ Document 2 $(\boldsymbol{d})$} \\
\hline Term & $\begin{array}{l}\text { Term } \\
\text { Count }\end{array}$ & Term & Term Count \\
\hline Dhaka & 3 & Dhaka & 5 \\
\hline is & 5 & is & 2 \\
\hline the & 4 & a & 4 \\
\hline capital & 1 & big & 1 \\
\hline of & 6 & population & 1 \\
\hline Bangladesh & 3 & country & 7 \\
\hline
\end{tabular}

TABLE. II. WEIGHT CALCULATION FROM DIFFERENT TEXTUAL DATASETS

\begin{tabular}{|l|l|l|l|l|l|l|}
\hline $\begin{array}{l}\text { Diabetes Dataset 1 } \\
\text { (Total 334 Patients) }\end{array}$ & \multicolumn{2}{l|}{$\begin{array}{l}\text { Diabetes Dataset 2 } \\
\text { (Total 203Patients) }\end{array}$} & Weight Calculation \\
\hline Attributes & Term Count & Term Frequency & Attributes & Term Count & Term Frequency & Average TF \\
\hline Hunger & 304 & 0.91 & Hunger & 165 & 0.81 & 0.86 \\
\hline Peeing more often & 278 & 0.83 & Peeing more often & 170 & 0.83 & 0.83 \\
\hline Dry Mouth & 311 & 0.93 & Dry Mouth & 173 & 0.85 & 0.89 \\
\hline Blurred Vision & 90 & 0.26 & Blurred Vision & 70 & 0.34 & 0.3 \\
\hline
\end{tabular}


2) Calculation of weight from numerical data: To generate the weight of an attribute from a dataset, the ration of the number of data samples containing that attribute and the total number of the sample data is calculated. Finally, the average weight of the attribute calculated from different datasets is measured. For example, we consider two Type-I diabetes patients' datasets where the first and second dataset both contain 334 and 203 diabetes-patients. There are four attributes (Hunger, Peeing, Dry mouth, and blurred vision) related to Type-I diabetes considering their corresponding affected patients as listed in Table II. Patients experiencing any of these four symptoms are represented and their corresponding term frequency is calculated by using equation 1. After calculating all the term frequency for both datasets, the average term frequency is calculated by using equation 3 .

\section{E. Weight Generation for Intermediate-layer Attributes}

Very often decision-makers need to consider some intermediate-level attributes to calculate the efficiency of a particular attribute in order to rank the decision attributes. Attributes at the intermediate level are not always seemed to be present directly in the datasets. However, their derivatives mostly determine their significance in decision-making tasks. Weight generation for the attributes of the intermediate level can be accomplished by using Cosine Similarity. Cosine Similarity measures the similarity between two non-zero vectors in a vector space model [17]. Suppose, $\alpha$ and $\beta$ are two non-zero vectors in a vector space model. So, cosines similarity between $\alpha$ and $\beta$ can be demonstrated as,

Cosine Similarity $=\frac{\alpha \cdot \beta}{\|\alpha\|\|\beta\|}$

To generate weights for the intermediate-level attributes, one of the non-zero vectors, $\alpha$ is constructed with the numeric values measured from the basic attributes of the decision problems which is always 1 . The other vector, $\beta$ is constructed with the Term Frequency of the attributes.

Weight of Attribute $A_{i}=\frac{\sum_{i=1}^{N} \alpha_{i} \beta_{i}}{\sqrt{\sum_{i=1}^{N} \alpha_{i}^{2}} \sqrt{\sum_{i=1}^{N} \beta_{i}^{2}}}$

For example, suppose, an intermediate-level attribute ' $\mathrm{I}$ ' has two derivatives ' $I_{1}{ }^{\prime}$ and ${ }^{\prime} I_{2}{ }^{\prime}$ and the TF of ${ }^{\prime} I_{1}{ }^{\prime}$ and ${ }^{\prime} I_{2}{ }^{\prime}$ are calculated as 0.9 and 0.87 respectively. So, the vectors $\alpha$ and $\beta$ for $A_{i}$ will be constructed as,

$\alpha=[1,1]$ and

$\beta=[0.9,0.87]$

Axiom-1: The value of the non-zero vectors used in the vector space model to generate the weight of intermediatelevel attributes in the architectural theory diagram of decision process is always considered 1. For example in equation 4, each parameter of vector $\alpha$ is considered 1 which can be represented as,

$\bar{\alpha}=[1,1,1, \ldots ., 1]$

In weight generation process explained in the earlier sections of this paper, one of the vector say $\alpha$ contains the frequency of the attributes. For example, to determine the weight of skin in the following decision problem, vector $\bar{\alpha}$ is constructed as $\bar{\alpha}=[1,1]$

Here, the weight of the skin is calculated based on the term frequency of purpura and edema in the historical datasets. To determine the importance of skin condition in the CKD diagnosis process, the skin condition is decomposed into its symptoms like edema and purpura (see Fig. 3). These two symptoms construct a vector. And the frequency of edema and purpura in the dataset constructs another vector. Here the value of the vector is always considered 1 to ensure the presence of edema and purpura in skin condition determination only once. If the value for edema would be considered 0 , the skin condition determination process would be accomplished without considering edema. On the other hand, if the value in the vector for edema is considered 2, edema will actually be considered twice to determine skin condition (see Fig. 4).

From the above three scenarios, it becomes clear that the value for $\bar{\alpha}$ should be $\bar{\alpha}=[1,1]$ todetermine the skin condition perfectly by avoiding the scenarios 2 and 3 .

Axiom-2: The weight of an attribute in decision-making process is always $0<w \leq 1$. Here $w$ is the weight of an attribute and the value of $\mathrm{w}$ is neither 0 nor less than 0 , $w<\neq 0$. Because if $w=0$, then the attribute has no impact on the decision-making process. Again the weight of an attribute can never be negative. If the weight of an attribute is negative then that attribute will have an adverse impact on decision process.

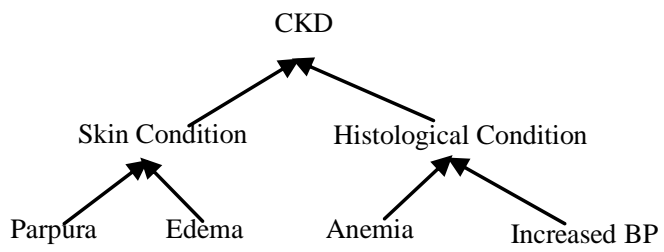

Fig. 3. Hierarchical Structure of CKD Diagnosis Problem.

$$
\text { Scenario-l: } \bar{\alpha}=[1,1] \quad\{\bar{\alpha}=[\text { edema, } \text { purpura }]\}
$$

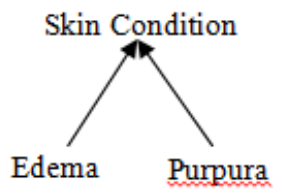

Scenario-2: $\bar{\alpha}=[0,1]$

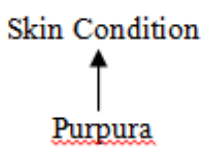

Scenario-3: $\bar{\alpha}=[2,1]$

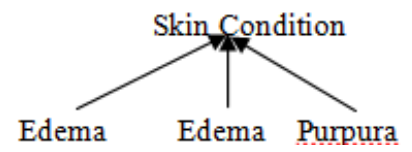

Fig. 4. Different Scenarios of Vector Construction Process. 


\section{NUMERICAL EXPERIMENT}

To conduct a numerical experiment, multiple sets of data from four different hospitals in Bangladesh have been collected. A total amount from 4000 CKD (Chronic Kidney Disease) patients has been taken into consideration in this experiment.

To make the experiment transparent and adaptable, the inputs and outputs of the experiment are expressed as follows:

$\mathrm{X}$ : Chronic Kidney Disease

$\mathrm{X}_{1}$ : General Condition

$\mathrm{X}_{2}$ : Gastrointestinal Condition

$\mathrm{X}_{3}$ : Skin Condition

$\mathrm{X}_{4}$ : Hematologic Condition

$\mathrm{X}_{11}$ : Pain on the side or mid to lower back

$\mathrm{X}_{12}$ : Fatigue

$\mathrm{X}_{13}$ : Mental Depression

$\mathrm{X}_{14}$ : Headaches

$\mathrm{X}_{21}$ : Vomiting

$\mathrm{X}_{22}$ : Loss Body Weight

$\mathrm{X}_{23}$ : Change Taste

$\mathrm{X}_{31}$ : Edema

$\mathrm{X}_{32}$ : Purpura

$\mathrm{X}_{41}$ : Blood in Urine

$\mathrm{X}_{42}$ : High Blood Pressure (HBP)

$\mathrm{X}_{43}$ : Loss of Appetite

$\mathrm{X}_{44}$ : Protein in Urine

In order to set weights to the CKD diagnosis attributes say symptoms of CKD, the presence of symptoms in datasets is carefully analyzed. To make the analysis convenient, the CKD diagnosis problem is organized in a hierarchical structure. Decision attributes are structured in a two layered architecture based on attribute nature and their impact on diagnosis process (see Fig. 5).

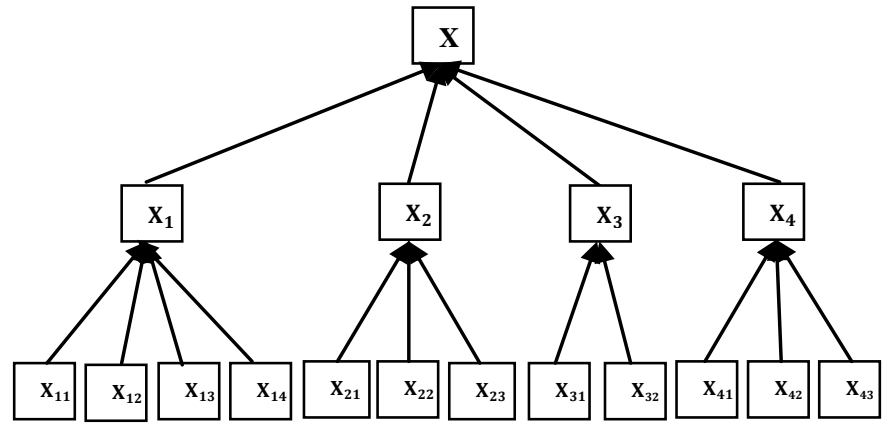

Fig. 5. Expression of Decision Problem in Hierarchical Structure.

\section{A. Weight Calculation for Root-Layer Attributes by using Average Term Frequency}

Term frequency calculates how many patients are experiencing a particular symptom over the total number of patients in a specific dataset. Based on how many times a symptom is present in a dataset, the weight for that symptom (attribute) is determined. The following algorithm provides a clear direction to the computation of the weights for the rootlayer attributes.

\section{Algorithm-1: TF calculation}

1. Start

2. Define

$$
\begin{gathered}
d \leftarrow \text { dataset } \\
t \leftarrow \text { rootlevelattributes } \\
n \leftarrow \text { numberofattributeappearsinadatset } \\
m \leftarrow \text { totalnumberofpatientinadatset } \\
T F \leftarrow \text { attribute's weight }
\end{gathered}
$$

3. Compute $T F \leftarrow \frac{n}{m}$

4. end

The following tables (Table. III-VI) show the weighs of different symptoms based on the TF calculation from different datasets. The TF of a symptom is calculated by using equation (1) and the weight is calculated by using equation (3).

Algorithm-2 in the next page provides an instruction for the implementation of equation (3) to generate weights for root-level attributes.

\section{Algorithm-2: Weight Calculation for Root-layer attributes}

Step 1: Start

Step 2: Define $i \leftarrow$ number of dataset

$$
\begin{gathered}
j \leftarrow \text { number of attributes } \\
T F \leftarrow \text { Term Frequency } \\
M \leftarrow \text { total number of } T F \\
N \leftarrow \text { average } T F
\end{gathered}
$$

Step 3: Set $T F \leftarrow 0$

$$
\begin{gathered}
M \leftarrow 0 \\
N \leftarrow 0 \\
a \leftarrow 1 \\
b \leftarrow 1
\end{gathered}
$$

Step 4: $M=M+T F$

Increase $a$ by 1

Go to Step 3 until $i=a$ and $j=b$

Step 5: Compute $N=\frac{M}{i}$

Print $N$

Step 6: End

For example, for a dataset as shown in Table III, the value of TF for $\boldsymbol{X}_{41}$ is calculated as $984 / 990=0.99$ where 990 is the total number of patients and 984 is the number of patients experiencing the symptoms "Blood in Urine". Therefore, the TF of the attribute $\boldsymbol{X}_{41}$ is 0.99 in case of dataset 1. However; the average $\mathrm{TF}$ of $X_{4 l}$ provides its actual weight which 0.97 as shown in Table VII. 
TABLE. III. CKD Dataset From BANGLADESh MEdicAl CollegEB (990 PATIENTS)

\begin{tabular}{|l|l|l|l|l|l|l|l|l|l|l|l|l|l|}
\hline & $\mathbf{X}_{\mathbf{1 1}}$ & $\mathbf{X}_{\mathbf{1 2}}$ & $\mathbf{X}_{\mathbf{1 3}}$ & $\mathbf{X}_{\mathbf{1 4}}$ & $\mathbf{X}_{\mathbf{2 1}}$ & $\mathbf{X}_{\mathbf{2 2}}$ & $\mathbf{X}_{\mathbf{2 3}}$ & $\mathbf{X}_{\mathbf{3 1}}$ & $\mathbf{X}_{\mathbf{3 2}}$ & $\mathbf{X}_{\mathbf{4 1}}$ & $\mathbf{X}_{\mathbf{4 2}}$ & $\mathbf{X}_{\mathbf{4 3}}$ & $\mathbf{X}_{\mathbf{4 4}}$ \\
\hline Attribute's Density & 770 & 970 & 550 & 223 & 406 & 601 & 333 & 379 & 421 & 984 & 980 & 801 & 705 \\
\hline Term Frequency & 0.77 & 0.97 & 0.55 & 0.22 & 0.41 & 0.6 & 0.33 & 0.38 & 0.42 & 0.99 & 0.98 & 0.8 & 0.71 \\
\hline
\end{tabular}

TABLE. IV. CKD DATASET From ShahEEdSuhrawardy Medical COLLEGE (1013 Patients)

\begin{tabular}{|l|l|l|l|l|l|l|l|l|l|l|l|l|l|}
\hline & $\mathbf{X}_{\mathbf{1 1}}$ & $\mathbf{X}_{\mathbf{1 2}}$ & $\mathbf{X}_{\mathbf{1 3}}$ & $\mathbf{X}_{\mathbf{1 4}}$ & $\mathbf{X}_{\mathbf{2 1}}$ & $\mathbf{X}_{\mathbf{2 2}}$ & $\mathbf{X}_{\mathbf{2 3}}$ & $\mathbf{X}_{\mathbf{3 1}}$ & $\mathbf{X}_{\mathbf{3 2}}$ & $\mathbf{X}_{\mathbf{4 1}}$ & $\mathbf{X}_{\mathbf{4 2}}$ & $\mathbf{X}_{\mathbf{4 3}}$ & $\mathbf{X}_{\mathbf{4 4}}$ \\
\hline Attribute's Density & 840 & 970 & 575 & 201 & 450 & 650 & 320 & 405 & 533 & 1001 & 998 & 905 & 695 \\
\hline Term Frequency & 0.82 & 0.95 & 0.56 & 0.19 & 0.44 & 0.64 & 0.31 & 0.4 & 0.52 & 0.98 & 0.98 & 0.89 & 0.68 \\
\hline
\end{tabular}

TABLE. V. CKD Dataset From Bangabandhu Medical College (997 Patients)

\begin{tabular}{|l|l|l|l|l|l|l|l|l|l|l|l|l|l|}
\hline & $\mathbf{X}_{\mathbf{1 1}}$ & $\mathbf{X}_{\mathbf{1 2}}$ & $\mathbf{X}_{\mathbf{1 3}}$ & $\mathbf{X}_{\mathbf{1 4}}$ & $\mathbf{X}_{\mathbf{2 1}}$ & $\mathbf{X}_{\mathbf{2 2}}$ & $\mathbf{X}_{\mathbf{2 3}}$ & $\mathbf{X}_{\mathbf{3 1}}$ & $\mathbf{X}_{\mathbf{3 2}}$ & $\mathbf{X}_{\mathbf{4 1}}$ & $\mathbf{X}_{\mathbf{4 2}}$ & $\mathbf{X}_{\mathbf{4 3}}$ & $\mathbf{X}_{\mathbf{4 4}}$ \\
\hline Attribute's Density & 699 & 965 & 507 & 245 & 423 & 629 & 400 & 405 & 467 & 981 & 987 & 790 & 695 \\
\hline Term Frequency & 0.7 & 0.96 & 0.5 & 0.24 & 0.42 & 0.63 & 0.4 & 0.4 & 0.46 & 0.98 & 0.98 & 0.79 & 0.69 \\
\hline
\end{tabular}

TABLE. VI. CKD Dataset From DhaKa Medical College (1104 Patients)

\begin{tabular}{|l|l|l|l|l|l|l|l|l|l|l|l|l|l|}
\hline & $\mathbf{X}_{\mathbf{1 1}}$ & $\mathbf{X}_{\mathbf{1 2}}$ & $\mathbf{X}_{\mathbf{1 3}}$ & $\mathbf{X}_{\mathbf{1 4}}$ & $\mathbf{X}_{\mathbf{2 1}}$ & $\mathbf{X}_{\mathbf{2 2}}$ & $\mathbf{X}_{\mathbf{2 3}}$ & $\mathbf{X}_{\mathbf{3 1}}$ & $\mathbf{X}_{\mathbf{3 2}}$ & $\mathbf{X}_{\mathbf{4 1}}$ & $\mathbf{X}_{\mathbf{4 2}}$ & $\mathbf{X}_{\mathbf{4 3}}$ & $\mathbf{X}_{\mathbf{4 4}}$ \\
\hline Attribute's Density & 809 & 1065 & 701 & 307 & 447 & 667 & 397 & 508 & 498 & 1050 & 1077 & 850 & 745 \\
\hline Term Frequency & 0.73 & 0.96 & 0.63 & 0.27 & 0.4 & 0.6 & 0.35 & 0.46 & 0.45 & 0.95 & 0.97 & 0.77 & 0.67 \\
\hline
\end{tabular}

TABLE. VII. Root-LeVEl ATtRibutes’'Weight CALCUlation

\begin{tabular}{|l|l|l|l|l|l|l|l|l|l|l|l|l|l|}
\hline Attributes & $\mathbf{X}_{\mathbf{1 1}}$ & $\mathbf{X}_{\mathbf{1 2}}$ & $\mathbf{X}_{\mathbf{1 3}}$ & $\mathbf{X}_{\mathbf{1 4}}$ & $\mathbf{X}_{\mathbf{2 1}}$ & $\mathbf{X}_{\mathbf{2 2}}$ & $\mathbf{X}_{\mathbf{2 3}}$ & $\mathbf{X}_{\mathbf{3 1}}$ & $\mathbf{X}_{\mathbf{3 2}}$ & $\mathbf{X}_{\mathbf{4 1}}$ & $\mathbf{X}_{\mathbf{4 2}}$ & $\mathbf{X}_{\mathbf{4 3}}$ & $\mathbf{X}_{\mathbf{4 4}}$ \\
\hline Attributes' weights & 0.75 & 0.96 & 0.56 & 0.23 & 0.42 & 0.62 & 0.35 & 0.41 & 0.46 & 0.97 & 0.97 & 0.81 & 0.69 \\
\hline
\end{tabular}

TABLE. VIII. WEIGHT CALCULATION OF INTERMEDIATE-LAYER ATtRIBUTES

\begin{tabular}{|c|c|c|c|c|c|c|c|c|c|c|c|c|c|}
\hline Intermediate-level Attributes & \multicolumn{4}{|l|}{$\mathbf{X}_{1}$} & \multicolumn{3}{|l|}{$\mathbf{X}_{2}$} & \multicolumn{2}{|l|}{$\mathbf{X}_{3}$} & \multicolumn{4}{|l|}{$\mathbf{X}_{4}$} \\
\hline Root-Level Attributes & $\mathbf{X}_{11}$ & $\mathbf{X}_{12}$ & $\mathbf{X}_{13}$ & $\mathbf{X}_{14}$ & $\mathbf{X}_{21}$ & $\mathbf{X}_{22}$ & $\mathbf{X}_{23}$ & $\mathbf{X}_{31}$ & $\mathbf{X}_{32}$ & $\mathbf{X}_{41}$ & $\mathbf{X}_{42}$ & $\mathbf{X}_{43}$ & $\mathbf{X}_{44}$ \\
\hline$\alpha$ & 1 & 1 & 1 & 1 & 1 & 1 & 1 & 1 & 1 & 1 & 1 & 1 & 1 \\
\hline $\boldsymbol{\beta}$ & 0.75 & 0.96 & 0.56 & 0.23 & 0.42 & 0.62 & 0.35 & 0.41 & 0.46 & 0.97 & 0.97 & 0.81 & 0.69 \\
\hline Attribute's weight & \multicolumn{4}{|l|}{0.92} & \multicolumn{3}{|l|}{0.97} & \multicolumn{2}{|l|}{0.35} & \multicolumn{4}{|l|}{0.99} \\
\hline
\end{tabular}




\section{B. Weight Calculation for Intermediate-Layer Attributes by using Cosine Similarity}

Attribute weights for intermediate layer are determined by the cosine similarity. Cosine similarity measures the cosine angle between two non-zero vectors. Intermediate layer attribute weight is calculated by the following algorithm shown in Table VIII. In Table VIII, the two non-zero vectors $\boldsymbol{\alpha}$ and $\boldsymbol{\beta}$ are defined according to the Section 3.3.

\section{Algorithm-3: Weight Calculation for Intermediate-layer attributes}

Step 1: Start

Step 2: Define $\mathrm{W} \leftarrow$ weight

Step 3: Set $d \leftarrow 0$

$$
\begin{aligned}
& n \leftarrow 0 \\
& e \leftarrow 0 \\
& f \leftarrow 0 \\
& \text { for all } j=1 \text { to } n \\
& \text { for all } i=1 \text { to } n
\end{aligned}
$$

Computed $\leftarrow a_{i} \times b_{i}$

Increase $i$ by 1

Compute $n=n+d$

$$
\text { End for }
$$

End for

Step 4: Set $m \leftarrow 0$

$$
\begin{gathered}
\text { for all } i=1 \text { to } n \\
m \leftarrow a_{i} \times a_{i} \\
e \leftarrow \operatorname{sqrt}(m)
\end{gathered}
$$

End for

Step 5: Set $m \leftarrow 0$

$$
\begin{gathered}
\text { for all } i=1 \text { to } n \\
m \leftarrow b_{i} \times b_{i} \\
f \leftarrow \operatorname{sqrt}(m)
\end{gathered}
$$

End for

$$
\text { Step 6:W }=\frac{n}{e \times f}
$$

Step 7: Print $\mathrm{W}$

Step 8: End

\section{RESUlt AND DISCUSSION}

Weight assignment process has been validated through some numerical experiments. For that, weights generated by the proposed system are compared to the benchmark results. The benchmark weights are calculated by analyzing a huge number of historical data by a group of domain experts. The accuracy of the proposed framework is found very close to the benchmark results.

To demonstrate that the proposed system performs better than any of the existing weight allocation approaches, some selected weight allocation methods have been experimented.

To analyze the performance of artificial neural network (ANN), the same input attributes and four datasets of the previous problem have been considered. In ANN, when input comes in the neural unit it is multiplied by a random weight of the corresponding node, and then the summation of the output of every node is performed. The final output comes out after transferring the current result into the sigmoid activation function for checking the current result against the threshold. To analyze the CKD problem, we consider a total of 12 attributes and feed the attribute values to the feedforward neural network with one hidden layer. The backpropagation algorithm sets the weights after 10 successful iterations.

On the other hand, some popular rule-based approaches like RIMER consider some random values as the attribute's initial weights which are later updated based on some thresholds. The domain experts manually set up the thresholds for making the weight updating process operational. To make a comparison among different weight allocation processes, the CKD diagnosis problem described earlier in this paper has been considered to generate the weighs by using the RIMER approach.

The other two prominent weight allocation methods- AHP and TOPSIS have also been utilized to generate weights for the attributed to a similar decision problem.

The Table IX provides a clear description of the performance level of different weighs assignment approaches. From the above table, it becomes very clear that TOPSIS and AHP methods result much differently than the other three methods. The ANN, RIMER and Proposed methodologies provide almost similar weights for almost all decision attributes. However, the optimal weight allocating framework can be selected by comparing the results of different methods against the benchmark results (see Fig. 6).

TABLE. IX. VisUALIZATION OF WEIGHT GENERATION VARIATIONS

\begin{tabular}{|l|l|l|l|l|l|}
\hline Attributes & AHP & TOPSIS & ANN & RIMER & $\begin{array}{l}\text { Proposed } \\
\text { Approach }\end{array}$ \\
\hline $\mathbf{X}_{\mathbf{1}}$ & 0.55 & 0.57 & 0.7 & 0.61 & 0.75 \\
\hline $\mathbf{X}_{\mathbf{2}}$ & 0.68 & 0.65 & 0.9 & 0.75 & 0.96 \\
\hline $\mathbf{X}_{\mathbf{3}}$ & 0.43 & 0.39 & 0.54 & 0.6 & 0.56 \\
\hline $\mathbf{X}_{\mathbf{4}}$ & 0.12 & 0.15 & 0.2 & 0.25 & 0.23 \\
\hline $\mathbf{X}_{\mathbf{5}}$ & 0.4 & 0.33 & 0.45 & 0.65 & 0.42 \\
\hline $\mathbf{X}_{\mathbf{6}}$ & 0.4 & 0.43 & 0.72 & 0.75 & 0.62 \\
\hline $\mathbf{X}_{\mathbf{7}}$ & 0.24 & 0.27 & 0.45 & 0.3 & 0.35 \\
\hline $\mathbf{X}_{\mathbf{8}}$ & 0.23 & 0.27 & 0.37 & 0.3 & 0.41 \\
\hline $\mathbf{X}_{\mathbf{9}}$ & 0.67 & 0.71 & 0.9 & 0.85 & 0.97 \\
\hline $\mathbf{X}_{\mathbf{1 0}}$ & 0.75 & 0.75 & 0.95 & 0.95 & 0.97 \\
\hline $\mathbf{X}_{\mathbf{1 1}}$ & 0.57 & 0.62 & 0.75 & 0.6 & 0.81 \\
\hline $\mathbf{X}_{\mathbf{1 2}}$ & 0.51 & 0.56 & 0.7 & 0.8 & 0.69 \\
\hline
\end{tabular}

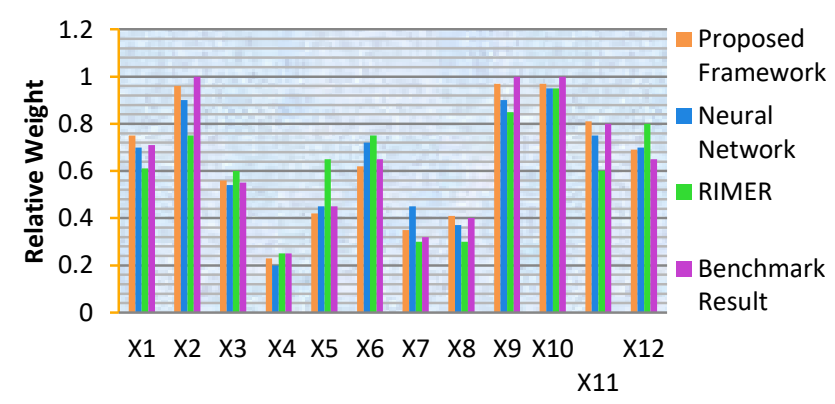

Fig. 6. Comparative Result of NN, RIMER, Benchmark Result with Proposed Framework. 
The above graph visualizes that, the proposed weight allocation method is very close to the benchmark results. Therefore, it can be strongly claimed that the proposed weight assignment approaches provide the optimum weights to the decision parameters.

\section{CONCLUSION}

Weight assignment is an important task in decisionmaking approaches. The solution of any decision problem mostly depends upon its attributes' weights. Unfortunately, there is no sophisticated framework for assigning weights to the decision parameters. So, decision-makers need to depend on human sensing factors to assign weights to the attributes that leads the decision-makers to a non-optimal solution. However, this paper proposes a weight setup method for multiple attribute decision problems where the weights will be calculated from some historical data without any engagement of the domain experts. The decision problem in this paper is represented in a hierarchical structure where the attribute layers are divided into intermediate and root layers. Weights for root-layer attributes are first determined by the term frequency of an individual dataset and then average term frequency is computed from the total number of datasets to find out the final weights. Cosine similarity is applied to compute the weights for intermediate-layer attributes which actually measures the similarity between two non-zero vectors. This can efficiently evade the unreasoning assessment values due to the lack of knowledge or partial experience of the experts. Additionally, a numerical experiment provided in this paper validates the effectiveness of the proposed methodology which can proficiently help the decision-makers assign the accurate weights to the decision attributes.

Funding: This research received no external funding.

Conflicts of Interest: The authors declare no conflict of interest.

\section{REFERENCES}

[1] W. Ho, X. Xu, and P. K. Dey, "Multi-criteria decision making approaches for supplier evaluation and selection: a literature review," European Journal of Operational Research, vol. 202, no. 1, pp. 16-24, 2010.

[2] Taho Yang, Yiyo Kuo, David Parker, and Kuan Hung Chen, "A Multiple Attribute Group Decision Making Approach for Solving Problems with the Assessment of Preference Relations," Mathematical Problems in Engineering, vol. 2015, 10 pages, 2015.
[3] Hongqiang Jiaoa and Shuangyou Wang, Multi-attribute decision making with dynamic weight allocation, Intelligent Decision Technologies 8 (2014) 225-230.

[4] JalilHeidaryDahooie, EdmundasKazimierasZavadskas, Amir SalarVanaki, Hamid Reza Firoozfar, MahnazLari\&ZenonasTurskis (2019) A new evaluation model for corporate financial performance using integrated CCSD and FCM-ARAS approach, Economic ResearchEkonomskaIstraživanja, 32:1, 1088-1113,2019.

[5] Quan Zhang and HongWeiXiu, "An Approach to Determining Attribute Weights Based on Integrating Preference Information on Attributes with Decision Matrix," Computational Intelligence and Neuroscience, vol. 2018, 8 pages, 2018.

[6] R. J. Ormerod and W. Ulrich, "Operational research and ethics: a literature review," European Journal of Operational Research, vol. 228, no. 2, pp. 291-307, 2013.

[7] C. L. Hwang and M. J. Lin, Group Decision Making Under Multiple Criteria: Methods and Applications, Springer, Berlin, Germany, 1987.

[8] J. R. Doyle, R. H. Green, and P. A. Bottomley, "Judging relative importance: Direct rating and point allocation are not equivalent," Org. Behav. Human Decis. Process., vol. 70, no. 1, pp. 65-72, Apr. 1997.

[9] R. Roberts and P. Goodwin, "Weight approximations in multi-attribute decision models," J. Multi Crit. Decis. Anal., vol. 11, no. 6, pp. 291303, Nov. 2002.

[10] C. L. Hwang and K. Yoon, Multiple Attribute Decision Making: Methods and Applications. Berlin, Germany: Springer, 1981.

[11] S. H. Zanakis, A. Solomon, N. Wishart, and S. Dublish, "Multi-attribute decision making: A simulation comparison of select methods," Eur. J. Oper. Res., vol. 107, no. 3, pp. 507-529, Jun. 1998.

[12] A. Charnes, W. W. Cooper, and E. Rhodes, "Measuring the efficiency of decision making units," Eur. J. Oper. Res., vol. 2, no. 6, pp. 429-444, Nov. 1978.

[13] W. D. Cook and M. Kress, "A data envelopment model for aggregatingpreference rankings,"Manag. Sci., vol. 36, no. 11, pp. 1302 1310, 1990.

[14] Z.-P. Fan, J. Ma, and Q. Zhang, "An approach to multiple attribute decision making based on fuzzy preference information on alternatives, "Fuzzy Sets. Syst., vol. 131, no. 1, pp. 101-106, Oct. 2002.

[15] D. Horsky and M. R. Rao, "Estimation of attribute weights frompreference comparisons,"Manag. Sci., vol. 30, no. 7, pp. 801822,Jul. 1984.

[16] Shu, Peng \& Ma, Jian \& Zhang, Quan. (2002). An approach to multiple attribute decision making based on fuzzy preference information on alternatives. Fuzzy Sets and Systems. 131. 101-106. 10.1016/S01650114(01)00258-5.

[17] Md Zahid Hasan, Shakhawat Hossain, Md. Arif Rizvee and Md. Shohel Rana, "Content based Document Classification using Soft Cosine Measure" International Journal of Advanced Computer Science and Applications(IJACSA), 10(4), 2019. 